\title{
Prevention of body weight loss and sarcopenia by a novel selective androgen receptor modulator in cancer cachexia models
}

\author{
MEGUMI MORIMOTO $^{1}$, KATSUJI AIKAWA ${ }^{2}$, TAKAHITO HARA ${ }^{1}$ and MASUO YAMAOKA ${ }^{1}$ \\ ${ }^{1}$ Oncology Drug Discovery Unit; ${ }^{2}$ Integrated Technology Research Laboratories, Pharmaceutical Research \\ Division, Takeda Pharmaceutical Company Limited, Fujisawa, Kanagawa 251-8555, Japan
}

Received April 6, 2017; Accepted September 7, 2017

DOI: $10.3892 / \mathrm{ol} .2017 .7200$

\begin{abstract}
Cancer cachexia is a syndrome that impairs the quality of life and overall survival of patients, and thus the effectiveness of anticancer agents. There are no effective therapies for cancer cachexia due to the complexity of the syndrome, and insufficient knowledge of its pathogenesis results in difficulty establishing appropriate animal models. Previously, promising results have been obtained in clinical trials using novel agents including the ghrelin receptor agonist anamorelin, and the selective androgen receptor modulator (SARM) enobosarm to treat cachexia in patients with cancer. The present study examined the pharmacological effects of SARM-2f, a novel non-steroidal small molecule SARM, in animal models. SARM-2f increased body and skeletal muscle weight without significantly increasing the weight of the seminal vesicles or prostates of the castrated male rats. In the mice with tumor necrosis factor $\alpha$-induced cachexia, SARM-2f and TP restored body weight, carcass weight, and food consumption rate. In the C26 and G361 cancer cachexia animal models, body and carcass weight, lean body mass, and the weight of the levator ani muscle were increased by SARM-2f and TP treatments. Tissue selectivity of SARM- $2 \mathrm{f}$ was also observed in these animal models. The results demonstrate the anabolic effects of SARM-2f in a cytokine-induced cachexia model and other cancer cachexia models, and suggest that SARM-2f may be a novel therapeutic option for cachexia in patients with cancer.
\end{abstract}

Correspondence to: Dr Takahito Hara, Oncology Drug Discovery Unit, Pharmaceutical Research Division, Takeda Pharmaceutical Company Limited, 26-1, Muraoka-Higashi 2-Chome, Fujisawa, Kanagawa 251-8555, Japan

E-mail: takahito.hara@takeda.com

Abbreviations: SARM, selective androgen receptor modulator; $\mathrm{TP}$, testosterone propionate; $\mathrm{TNF} \alpha$, tumor necrosis factor alpha; $\mathrm{BB}$, benzyl benzoate; LBM, lean body mass

Key words: cancer cachexia, body weight loss, androgen, selective androgen receptor modulator, sarcopenia, anorexia

\section{Introduction}

Cancer cachexia is defined as a multifactorial disorder associated with an ongoing loss of skeletal muscle mass with or without loss of fat mass, which leads to a progressive functional impairment that cannot be fully reversed with conventional nutritional supplementation (1). The symptoms appear in up to $80 \%$ of patients with cancer and account for at least $20 \%$ of cancer-associated deaths $(1,2)$. Cancer cachexia has been reported to be caused by nutritional deficiencies, metabolic disorders, and inflammatory disorders (3-6). Presently, however, there is no approved therapy for the treatment or prevention of cancer cachexia. Therefore, an effective therapy for cancer cachexia is an unmet medical need. There are several potential therapeutic approaches for cancer cachexia (7-10). Androgen is a steroid hormone with multiple physiological functions including promotion of growth hormone release, appetite stimulation, anabolic actions, stimulatory effects on the central nervous system (CNS), and regulation of energy homeostasis. Low plasma androgen levels are often observed in patients with terminal cancer $(11,12)$. Therefore, androgen-based treatments might be a potential therapy for cancer cachexia. However, testosterone replacement therapy has unavoidable side effects such as myocardial infarction, heart failure, stroke, depression, and aggression $(13,14)$.

In a previous study, we reported the 4-(pyrrolidin-1-yl) benzonitrile derivative as a selective androgen receptor modulator (SARM)-1c (15). Unlike testosterone, SARM-1c exhibited anabolic effects on the levator ani muscles without excessively increasing prostate weight in rat Hershberger assays (15). We further improved the pharmacokinetic (PK) profile by modifying the structure and identified $4-[(2 \mathrm{~S}, \mathrm{~S})$ -2-ethyl-3-hydroxy-5-oxopyrrolidin-1-yl]-2-(trifluoromethyl) benzonitrile (SARM-2f) as a novel SARM (Fig. 1). In the present study, we examined the effects of SARM-2f on the weight of skeletal muscles (i.e., the gastrocnemius and soleus muscles), body weight, body composition, and anorexia in a castrated rat model and three cancer cachexia models.

\section{Materials and methods}

Ethical statement. The animal protocol for this study was approved by the Animal Ethics Committee of Takeda Pharmaceutical Company Ltd. In addition, all procedures were 
performed according to protocols approved by the Institutional Animal Care and Use Committee of the Pharmaceutical Research Division of Takeda Pharmaceutical Company Limited. The protocols were in accordance with the ethical standards laid down in the 1964 Declaration of Helsinki and its later amendments.

Animals and compounds. CD (Sprague-Dawley, SD, IGS) rats were purchased from Charles River Laboratories Japan (Yokohama, Japan). F344 immunodeficient rats (5- or 15-week-old) and BALB/c mice (5- or 6-weeks-old) were obtained from CLEA Japan, Inc. (Tokyo Japan). The animals were maintained under a 12-h light/dark cycle at a constant temperature of $23 \pm 2^{\circ} \mathrm{C}$ while food and water were provided ad libitum. SARM-2f (4-((2S, 3S)-2-ethyl-3-hydroxy-5-oxopyrrol idin-1-yl)-2-(trifluoromethyl)benzonitrile) was synthesized by Takeda Pharmaceutical Company Ltd., and suspended in $0.5 \%$ methylcellulose. Tumor necrosis factor (TNF)- $\alpha$ (WAKO Pure Chemical Industries, Ltd., Osaka, Japan) was dissolved in saline (Otsuka, Tokushima, Japan), whereas testosterone propionate (TP; Tokyo Chemical Industry, Tokyo, Japan) was dissolved in $20 \%$ benzyl benzoate and $80 \%$ corn oil (BBoil).

Castrated rat model. Mature 12-week-old male SD (ID) IGS rats were castrated under isoflurane anesthesia. Then, 8 weeks later, they were divided into six groups $(n=10)$ based on body weight (day 0). The animals were then administered the vehicle $(0.5 \%$ methylcellulose, orally, p.o.; once daily, QD) and BBoil (subcutaneously, s.c., thrice weekly), SARM-2f (0.3, 3, or $30 \mathrm{mg} / \mathrm{kg}$, p.o., QD) and BBoil (s.c., thrice weekly), or TP (2.5 mg/kg, s.c., thrice weekly) and $0.5 \%$ methylcellulose (p.o., QD) for 4 weeks. Body weight was measured twice weekly, whereas the body composition was determined using nuclear magnetic resonance spectroscopy (EchoMRI-700, Echo Medical Systems, Houston, TX, USA) on day 0 and 27. On day 28 , the skeletal muscles and sex accessory organs were removed and weighed. The organs were immersed in saline until weighed to prevent them from drying out.

TNF $\alpha$-induced cachexia mouse model. Seven-week-old $\mathrm{BALB} / \mathrm{c}$ male mice were castrated under anesthesia (day 0). Intraperitoneal (i.p.) injections of $\mathrm{TNF} \alpha(5 \mu \mathrm{g} /$ day twice daily, BID) were administered to the mice starting from day 7 and continued for 2 weeks. The animals were simultaneously treated with SARM-2f (10 or $30 \mathrm{mg} / \mathrm{kg}$, p.o., BID) and BBoil (s.c., QD) or TP (30 mg/kg, s.c., QD) and $0.5 \%$ methylcellulose (p.o., BID). The body weight and amount of food consumed were measured twice weekly. In addition, carcass weight (i.e., the weight of the mouse after removal of all internal organs) and wet organ weight were measured at the end of the study.

G361 cancer cachexia model. Fifteen-week-old F344 immunodeficient rats were used for this study. Under isoflurane anesthesia, 5x10 6361 human melanoma cells (American Tissue Culture Collection, ATCC, Manassas, VA, USA) were implanted subcutaneously into one flank of each rat (day 0). The rats were then administered the vehicle [0.5\% methylcellulose (p.o., QD)] and BBoil (s.c., thrice weekly), SARM-2f (3 mg/kg, p.o., QD) and BBoil (s.c., thrice weekly), or TP $(2.5 \mathrm{mg} / \mathrm{kg}$, s.c., thrice weekly) and $0.5 \%$ methylcellulose (p.o., QD) for
4 weeks starting from day 7 . The tumor size and body weight were measured twice a week. The tumor volume (expressed in $\mathrm{mm}^{3}$ ) was calculated using the formula $0.5 \mathrm{x} \mathrm{a} \mathrm{x} \mathrm{b}$, where ' $\mathrm{a}$ ' is the largest diameter, and ' $b$ ' is largest diameter perpendicular to 'a.' Lean body mass was measured using an EchoMRI-700 on days 7 and 35. The muscles were removed and measured at the end of the study.

C26 cancer cachexia model. Six-week-old male BALB/c mice were used for this model. Briefly, $5 \times 10^{5} \mathrm{C} 26$ mouse carcinoma cells were subcutaneously implanted into one flank of each mouse on the day of castration (day 0). The mice were then administered the vehicle [0.5\% methylcellulose (p.o., BID)] and BBoil (s.c., QD), SARM-2f (10, 30, or $100 \mathrm{mg} / \mathrm{kg}$, p.o., BID) and BBoil (s.c., QD), or TP (30 mg/kg, s.c., QD) and $0.5 \%$ methylcellulose (p.o., BID) for 2 weeks starting from day 11 . The tumor size and body weight were measured twice weekly. The tumor volume was expressed as described above. Carcass, muscle, and organ weights were measured at the end of the study.

Statistical analysis. Differences between the control and treatment groups were analyzed using the Student's t-test, Williams' test, or Dunnett's test.

\section{Results}

Effects of SARM-2f on body weight and skeletal muscles of castrated rats. The effects of SARM-2f on castrated rats are presented in Fig. 2. Castration decreased the body weight, lean body mass, and the weight of the levator ani muscle, prostate, and seminal vesicle (Fig. 2A, B, D, G, and H); however, it did not influence fat mass or the weight of the gastrocnemius or soleus muscles (Fig. 2C-F). Treatment with SARM-2f at doses of $0.3,3$, and $30 \mathrm{mg} / \mathrm{kg}$ significantly increased the body weight gain (2.2-fold, 2.2-fold, and 2.1-fold, respectively), lean body mass (5.4-fold, 6.3-fold, and 7.2-fold, respectively), and weight of the levator ani (2.3-fold, 2.6-fold, and 2.6-fold, respectively), gastrocnemius (1.1-fold, 1.02-fold, and 1.06-fold, respectively), and soleus (1.1-fold, 1.1-fold, and 1.1-fold, respectively) muscles of the castrated rats (Fig. 2A, B, and D-F). However, SARM-2f treatment did not influence the fat mass (Fig. 2C). Treatment with TP at a dose of $2.5 \mathrm{mg} / \mathrm{kg}$ increased the levator ani muscle weight (2.6-fold) but not the body weight (Fig. 2A and D). The TP treatment significantly reduced the fat mass (Fig. 2C). Treatment with SARM-2f at doses of $0.3,3$, and $30 \mathrm{mg} / \mathrm{kg}$ increased the weights of the prostate (3.2-fold, 4.8-fold, and 6.4-fold, respectively) and seminal vesicle (9.8-fold, 19-fold, and 28-fold, respectively) in a dose-dependent manner in the castrated rats (Fig. 2G and H). However, the prostate and seminal vesicle weights of the SARM-2f-treated castrated rats were lower than the respective weights of the non-castrated group (Fig. $2 \mathrm{G}$ and $\mathrm{H})$. In contrast, TP increased the weights of the prostate (11-fold) and seminal vesicle (42-fold) in the castrated rats, whichwere higher than the respective weights in the non-castrated group (Fig. 2G and H). These results demonstrate the anabolic effect and tissue specificity of SARM-2f.

Recovery of food intake rate and body weight by SARM-2f in TNFa-induced cachexia mouse model. We examined the 


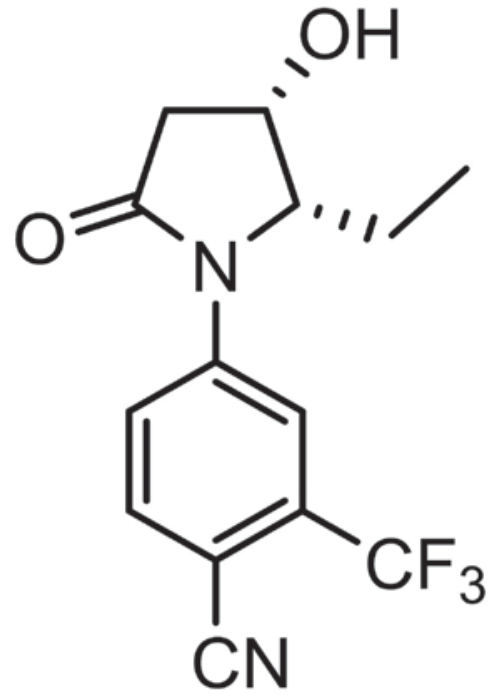

Figure 1. Chemical structure of selective androgen receptor modulator (SARM)-2f.

effects of SARM-2f on inflammation-related cachexia in the $\mathrm{TNF} \alpha$-induced cachexia mouse model. TNF $\alpha$ caused body weight loss in castrated mice (Fig. 3A). However, SARM-2f at doses of 10 and $30 \mathrm{mg} / \mathrm{kg}$ as well as TP at $30 \mathrm{mg} / \mathrm{kg}$ increased the body weight in the TNF $\alpha$-treated mice (Fig. 3A). SARM-2f at doses of 10 and $30 \mathrm{mg} / \mathrm{kg}$ increased the carcass weight (1.1-fold and 1.1-fold, respectively), and food consumption rate (1.1-fold and 1.1-fold, respectively) in TNF $\alpha$-treated mice (Fig. 3B and C). TP increased the carcass weight (1.1-fold) and food consumption rate (1.1-fold) in TNF $\alpha$-treated mice (Fig. 3B and C).

Prevention of skeletal muscle and bodyweight loss by SARM-2f in G361-tumor-bearing rats. The growth of the G361 human melanoma cells that were subcutaneously grafted into the F344 immunodeficient rats caused progressive body weight loss and a decrease in lean body mass in the rats (Fig 4A and B). Treatment with SARM-2f or TP did not change the body weight and lean body mass (Fig. 4A and B). However, both SARM-2f and TP treatment increased the weight of the levator ani muscle (1.6-fold and 1.7-fold, respectively) (Fig. 4C). TP but not SARM-2f increased the prostate and seminal vesicle weights (Fig. 4D and E). The tumor growth was unaffected by either treatment (Fig. 4F).

Prevention of body weight loss by SARM-2f in C26-tumor-induced cachexia mouse model. The mouse allograft model of $\mathrm{C} 26$ adenocarcinoma is a well-established model for investigating body weight loss caused by cancer cachexia (16-18). We found that SARM-2f at doses of 10, 30, and $100 \mathrm{mg} / \mathrm{kg}$ increased the body (1.1-fold, 1.1-fold, and 1.1-fold, respectively), carcass (1.1-fold, 1.1-fold, and 1.1-fold, respectively), and levator ani muscle weights (3.3-fold, 2.9-fold, and 3.8-fold respectively) in the castrated mice (Fig. 5A-C). No effect was observed on the ventral prostate weight at a dose of $10 \mathrm{mg} / \mathrm{kg}$ (Fig. 5D). Meanwhile, TP increased the body weight (1.2-fold), carcass weight (1.2-fold), and the weights of the levator ani muscle (4.6-fold), ventral prostate (3.8-fold), and seminal vesicle (19-fold) (Fig. 5A-E).
Neither SARM-2f nor TP had any effects on tumor growth in this model (Fig. 5F).

\section{Discussion}

We observed that SARM-2f increased the weights of the gastrocnemius and soleus muscles in the castrated rats. In addition, it prevented body weight loss in the TNF $\alpha$-treated model and the $\mathrm{C} 26$ tumor model, and muscle wasting in the $\mathrm{C} 26$ and G361 tumor models. Moreover, it improved anorexia in the $\mathrm{TNF} \alpha$-treated model. It has been reported that testosterone increases gastrocnemius muscle weight in rats (19). However, our data showed that a $2.5 \mathrm{mg} / \mathrm{kg}$ TP dose increased the weights of the levator ani muscle and the prostate but had no effects on the weights of the gastrocnemius and soleus muscles without forced exercise in the castrated male rat model.

The anabolic effect of testosterone on the skeletal muscles is controversial and considered dose-dependent (20). Interestingly, SARM-2f increased the weights of both the gastrocnemius and soleus muscles in our study. Currently, the difference between the anabolic effects of TP and those of SARM-2f is unclear. However, the tissue distribution and tissue-selective gene expression profiles of testosterone may differ from those of SARM-2f and, therefore, the effects of the two drugs on balancing the synthesis and breakdown of skeletal muscle protein may differ.

Several mechanisms have been proposed to explain the effects of SARM-2f on body weight and body composition in cancer cachexia models. One possible mechanism is the direct activation of androgen receptors in the muscles while another is likely mediated by activation of androgen receptors in other tissues such as CNS, which may affect behavior, amount of exercise, and appetite (21). We previously demonstrated that at a dose of $0.5 \mathrm{mg} / \mathrm{kg}$ QD (p.o.), SARM-2f enhanced the sexual behavior of castrated rats (22). In the present study, SARM-2f increased the food intake of mice with TNF $\alpha$-induced cachexia. These findings suggest that SARM-2f might have CNS effects that improve cachexia.

The other possible mechanism is the suppression of inflammation, and inflammatory cytokines such as TNF $\alpha$, interleukin (IL)-1, and IL-6 have been reported to be involved in cancer cachexia $(1,5,23,24)$. Moreover, nandrolone decanoate, an anabolic steroidal androgen, has been shown to regulate inflammatory processes in skeletal muscles (2). Therefore, further studies are required to elucidate the mechanism underlying the effect of SARM-2f on cancer cachexia.

The causes of cancer cachexia are poorly understood because of its heterogeneity, which possibly stems from the heterogeneity and complexity of cancer. Several cancer cachexia models such as ectopic or orthotopic transplanted xenograft and allograft models have been reported (17). However, no animal model completely mimics the complexity of human cancer cachexia (17). Several factors such as diversity of cancer, use of chemotherapy, exercise, or nutritional support should be taken into consideration, and suitable experimental models reflecting the clinical situation should be used for accurate evaluation of compounds for the treatment of cancer cachexia.

Despite evidence of the androgen-induced improvement of muscle wasting and anorexia, androgen replacement therapy 

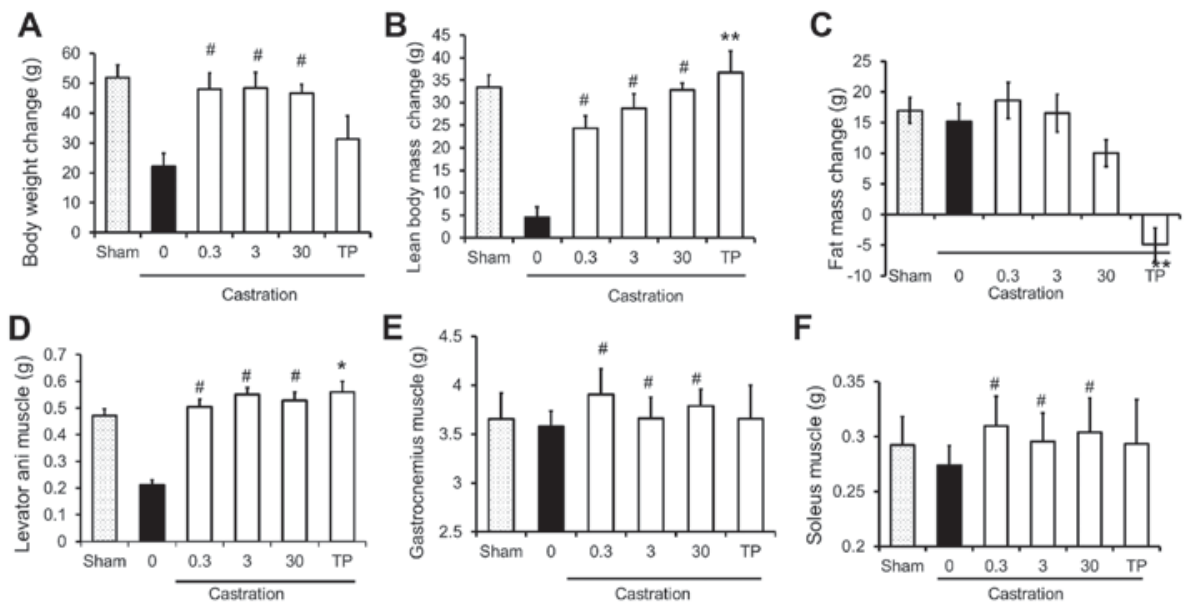

$\mathbf{F}$

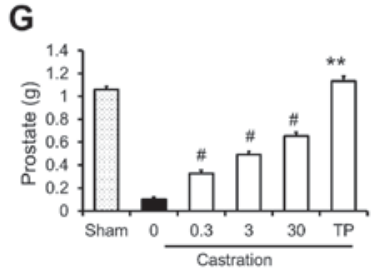

H
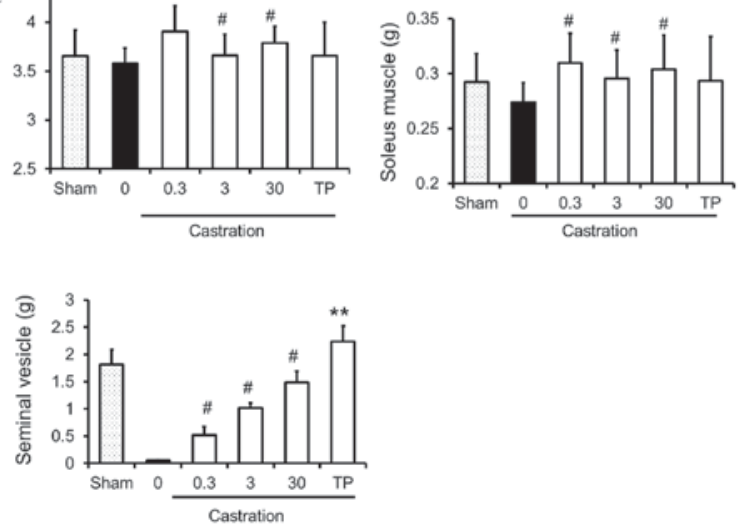

Figure 2. Anabolic effects of selective androgen receptor modulator (SARM)-2f in castrated rats. Changes of body weight (A), lean body mass (B), and fat mass (C) between day 0 and day 26 or 27 in sham-, SARM-2f-, and testosterone propionate (TP)-treated rats. Weights of (D) levator ani muscle, (E) gastrocnemius muscle, $(\mathrm{F})$ soleus muscle, $(\mathrm{G})$ prostate, and $(\mathrm{H})$ seminal vesicle were measured at the end of the study. Data are mean \pm standard error of the mean (SEM, $\mathrm{n}=10$ ). ${ }^{.} \mathrm{P}<0.025$ (Williams' test) compared to vehicle control group; ${ }^{*} \mathrm{P}<0.05$ and ${ }^{* *} \mathrm{P}<0.01$ (Student's $\mathrm{t}$-test) compared to vehicle control group.

A

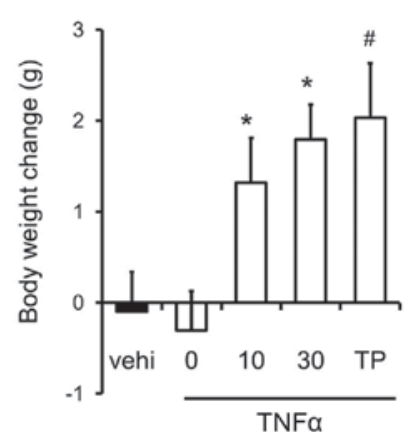

B

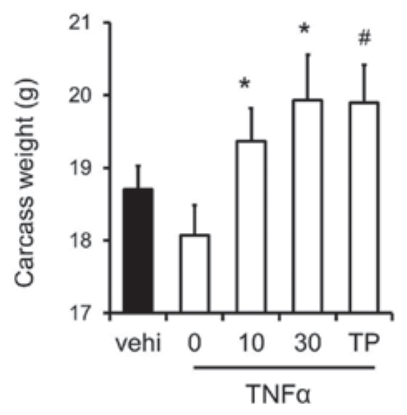

C

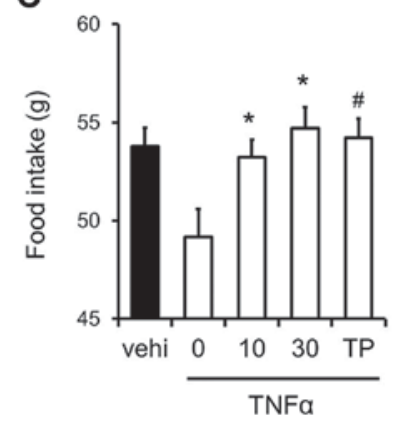

Figure 3. Recovery of body weight and food intake in mice with tumor necrosis factor (TNF)- $\alpha$-induced cachexia. (A) Body weight change, (B) carcass weight, and (C) food intake in mice treated with vehicle, selective androgen receptor modulator (SARM)-2f (10 or 30 mg/kg; oral, p.o.; twice daily BID), or testosterone propionate (TP, $30 \mathrm{mg} / \mathrm{kg}$, subcutaneous, s.c.; daily, QD). Body weight change and food intake indicate the change between day 7 and day 21 . Data are mean \pm standard error of the mean (SEM, $n=9) .{ }^{~} \mathrm{P}<0.05$ (Dunnett's test) compared to TNF $\alpha$-treated castrated mice. ${ }^{*} \mathrm{P}<0.05$ (Student's t-test) compared to the TNF $\alpha$-treated castrated mice.

is not a standard treatment for cancer cachexia (25-27). Testosterone may be a viable therapeutic option for cancer cachexia. However, clinical trials with large sample sizes are needed to confirm whether testosterone is an efficacious treatment for improving body composition, physical function, quality of life, and cancer survival of patients with cachexia. A double-blind, placebo-controlled, interventional study investigating this is currently in progress (NCT00878995). The major concern associated with testosterone use is the unavoidable side effects such as cardiovascular disorders $(14,15)$, which may be partly attributable to estrogen derived from testosterone $(13,28)$. However, SARM-2f is not a substrate of aromatase, which is the enzyme that converts testosterone to estradiol. Therefore, unlike testosterone therapy, treatment with SARM-2f will not result in the unwanted side effects of estrogen.

SARMs have been widely investigated in preclinical animal models of muscle wasting, hypogonadism, osteoporosis, and other conditions (29-34). Enobosarm is one of the SARMs developed for treating muscle-wasting diseases. It showed tissue-selectivity similar to SARM-2f in the Hershberger assay (35). To the best of our knowledge, enobosarm has not been reported to be effective in cancer cachexia models; however, it has been reported to increase lean body mass and improve muscle function in patients with non-small cell lung carcinoma (NSCLC) in a Phase III clinical trial $(36,37)$. 
A

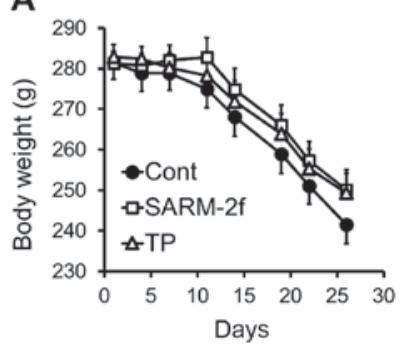

D

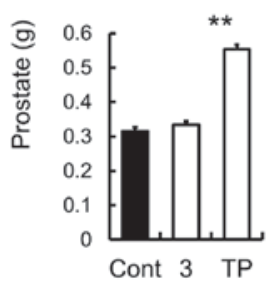

B

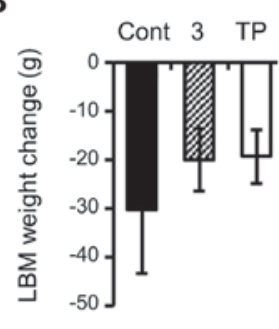

C

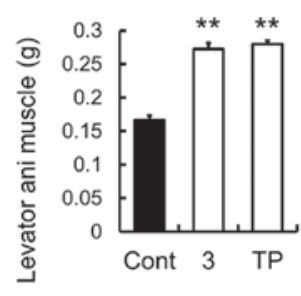

F

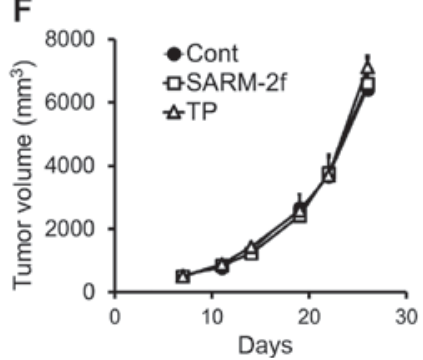

Figure 4. Body and muscle weight in G361-tumor-bearing rats (A) Body weight was measured twice weekly. (B) Lean body mass (LBM) change between day 7 and day 35 by treatment with vehicle, selective androgen receptor modulator (SARM)-2f (3 mg/kg; oral, p.o.; daily, QD), or testosterone propionate (TP, $2.5 \mathrm{mg} / \mathrm{kg}$, subcutaneous, s.c.; thrice weekly). Weights of (C) levator ani muscle, (D) prostate, and (E) seminal vesicle were measured at the end of the study. (F) Tumor volume was measured twice weekly as described in the methods. Data are mean \pm standard error of the mean (SEM, $\mathrm{n}=9-10)$. ${ }^{*}<0.05$ (Student's t-test) compared to the vehicle control group.

A

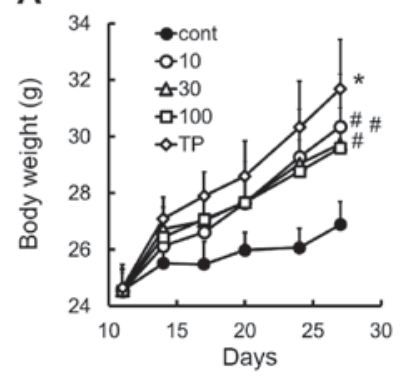

D

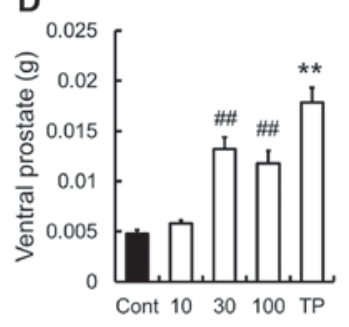

B

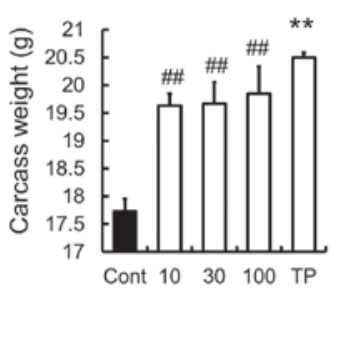

E

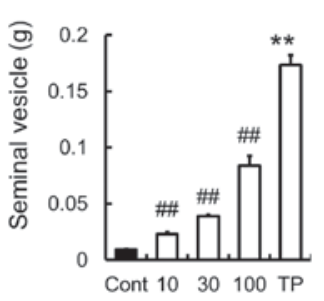

C

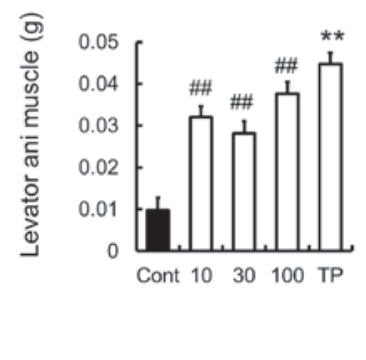

$\mathbf{F}$

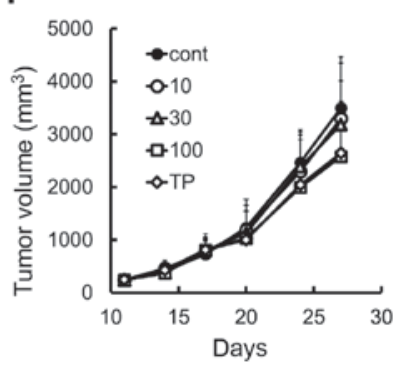

Figure 5. Prevention of body weight loss in C26 cancer cachexia model (A) Body weight was measured twice weekly. (B) Carcass weight and the weights of (C) levator ani muscle, (D) ventral prostate, and (E) seminal vesicle were measured after treatment with selective androgen receptor modulator (SARM)-2f (10, 30 , or $100 \mathrm{mg} / \mathrm{kg}$; oral, p.o.; twice daily, BID) or testosterone propionate (TP, $30 \mathrm{mg} / \mathrm{kg}$, subcutaneous, s.c.; once daily, QD) on day 27. (F) Tumor volume was measured twice weekly as described in methods. Data are mean \pm standard error of the mean $(\mathrm{SEM}, \mathrm{n}=7)$. ${ }^{\#} \mathrm{P}<0.05$ and ${ }^{\# \#} \mathrm{P}<0.01$ (Dunnett's test) compared to control group. ${ }^{* *} \mathrm{P}<0.01$ (Student's t-test) compared to control group.

However, in another clinical trial on NSCLC, enobosarm did not improve stair climb power in patients with cachexia $(36,37)$. Therefore, larger clinical trials should be conducted to confirm the therapeutic potential of enobosarm.

In conclusion, we demonstrated that SARM-2f increased body and skeletal muscle weight without excessively stimulating sex accessory organs. It also improved anorexia in rat and mouse cachexia models, suggesting that it may be an effective therapeutic option for treating cachexia in patients with cancer.

\section{Acknowledgements}

We would like to thank Jin Kouno and Tsuneo Masaki for providing technical assistance at Takeda Pharmaceutical 
Company Limited. The present study was funded by Takeda Pharmaceutical Company Ltd.

\section{References}

1. Fearon K, StrasserF, Anker SD, Bosaeus I,Bruera E, Fainsinger RL, Jatoi A, Loprinzi C, MacDonald N, Mantovani G, et al: Definition and classification of cancer cachexia: An international consensus. Lancet Oncol 12: 489-495, 2011.

2. Argilés JM, Busquets S, Stemmler B and López-Soriano FJ: Cancer cachexia: Understanding the molecular basis. Nat Rev Cancer 14: 754-762, 2014.

3. Fearon KC, Glass DJ and Guttridge DC: Cancer cachexia: Mediators, signaling, and metabolic pathways. Cell Metab 16: 153-166, 2012.

4. Cooper C, Burden ST, Cheng H and Molassiotis A: Understanding and managing cancer-related weight loss and anorexia: Insights from a systematic review of qualitative research. J Cachexia Sarcopenia Muscle 6: 99-111, 2015.

5. Tuca A, Jimenez-Fonseca P and Gascón P: Clinical evaluation and optimal management of cancer cachexia. Crit Rev Onco Hematol 88: 625-636, 2013.

6. Porporato PE: Understanding cachexia as a cancer metabolism syndrome. Oncogenesis 5: e200, 2016.

7. Coss CC, Bohl CE and Dalton JT: Cancer cachexia therapy: A key weapon in the fight against cancer. Curr Opin Clin Nutr Metab Care 14: 268-273, 2011.

8. Dodson S, Baracos VE, Jatoi A, Evans WJ, Cella D, Dalton JT and Steiner MS: Muscle wasting in cancer cachexia: Clinical implications, diagnosis, and emerging treatment strategies. Annu Rev Med 62: 265-279, 2011.

9. Madeddu C, Mantovani G, Gramignano G and Macciò A Advances in pharmacologic strategies for cancer cachexia. Expert Opin Pharmacother 16: 2163-2177, 2015.

10. Molfino A, Amabile MI, Rossi Fanelli F and Muscaritoli M: Novel therapeutic options for cachexia and sarcopenia. Expert Opin Biol Ther 16: 1239-1244, 2016.

11. Strasser F, Palmer JL, Schover LR, Yusuf SW, Pisters K, Vassilopoulou-Sellin R, DeGracia B, Willey JS and Bruera E: The impact of hypogonadism and autonomic dysfunction on fatigue, emotional function, and sexual desire in male patients with advanced cancer: A pilot study. Cancer 107: 2949-2957, 2006.

12. Burney BO, Hayes TG, Smiechowska J, Cardwell G, Papusha V, Bhargava P, Konda B, Auchus RJ and Garcia JM: Low testosterone levels and increased inflammatory markers in patients with cancer and relationship with cachexia. J Clin Endocrino Metab 97: E700-E709, 2012.

13. Basaria S, Coviello AD, Travison TG, Storer TW, Farwell WR, Jette AM, Eder R, Tennstedt S, Ulloor J, Zhang A, et al: Adverse events associated with testosterone administration. N Engl J Med 363: 109-122, 2010.

14. Vigen R, O'Donnell CI, Barón AE, Grunwald GK, Maddox TM, Bradley SM, Barqawi A, Woning G, Wierman ME, Plomondon ME, et al: Association of testosterone therapy with mortality, myocardial infarction, and stroke in men with low testosterone levels. JAMA 10: 1829-1836, 2013.

15. Aikawa K, Miyawaki T, Hitaka T, Imai YN, Hara T, Miyazaki J, Yamaoka M, Kusaka M, Kanzaki N, Tasaka A, et al: Synthesis and biological evaluation of novel selective androgen receptor modulators (SARMs). Part I. Bioorg Med Chem 23: 2568-2578, 2015.

16. Dwarkasing JT, Boekschoten MV, Argilès JM, van Dijk M, Busquets S, Penna F, Toledo M, Laviano A, Witkamp RF and van Norren K: Differences in food intake of tumor-bearing cachectic mice are associated with hypothalamic serotonin signaling. J Cachexia Sarcopenia Muscle 6: 84-94, 2015.

17. Ballarò R, Costelli P and Penna F: Animal models for cancer cachexia. Curr Opin Support Palliat Care 10: 281-287, 2016.

18. Talbert EE, Metzger GA, He WA and Guttridge DC: Modeling human cancer cachexia in colon 26 tumor-bearing adult mice. J Cachexia Sarcopenia Muscle 5: 321-328, 2014.

19. Ustünel I, Akkoyunlu G and Demir R: The effect of testosterone on gastrocnemius muscle fibres in growing and adult male and female rats: A histochemical, morphometric and ultrastructural study. Anat Histol Embryol 32: 70-79, 2003.

20. Urban RJ: Growth hormone and testosterone: Anabolic effects on muscle. Horm Res Paediatr 76 (Suppl 1): S81-S83, 2011.
21. De Gendt K and Verhoeven G: Tissue- and cell-specific functions of the androgen receptor revealed through conditional knockout models in mice. Mol Cell Endocrinol 352: 13-25, 2012.

22. Aikawa K, Asano M, Ono K, Habuka N, Yano J, Wilson K, Fujita H, Kandori H, Hara T, Morimoto M, et al: Synthesis and biological evaluation of novel selective androgen receptor modulators (SARMs) Part III: Discovery of 4-(5-oxopyrrolidine-1-yl) benzonitrile derivative $2 \mathrm{f}$ as a clinical candidate. Bioorg Med Che 25: 3330-3349. 2017.

23. Argiles JM, Lopez-Soriano FJ and Busquets S: Counteracting inflammation: A promising therapy in cachexia. Crit Rev Oncog 17: 253-262, 2012

24. Thompson RW, McClung JM, Baltgalvis KA, Davis JM and Carson JA: Modulation of overload-induced inflammation by aging and anabolic steroid administration. Exp Gerontol 41: 1136-1148, 2006.

25. Srinath R and Dobs A: Enobosarm (GTx-024, S-22): A potential treatment for cachexia. Future Oncol 10: 187-194, 2014.

26. Dillon EL, Basra G, Horstman AM, Casperson SL, Randolph KM, Durham WJ, Urban RJ, Diaz-Arrastia C, Levine L, Hatch SS, et al: Cancer cachexia and anabolic interventions: A case report. J Cachexia Sarcopenia Muscle 3: 253-263, 2012.

27. Del Fabbro E, Garcia JM, Dev R, Hui D, Williams J, Engineer D, Palmer JL, Schover L and Bruera E: Testosterone replacement for fatigue in hypogonadal ambulatory males with advanced cancer: A preliminary double-blind placebo-controlled trial. Support Care Cancer 21: 2599-2607, 2013.

28. de Vooqt HJ, Smith PH, Pavone-Macaluso M, de Pauw M and Suciu S: Cardiovascular side effects of diethylstilbestrol, cyproterone acetate, medroxyprogesterone acetate and estramustine phosphate used for the treatment of advanced prostatic cancer: Results from European Organization for Research on Treatment of Cancer Trials 30761 and 30762. J Urol 135: 303-307, 1986

29. Ponnusamy S, Sullivan RD, Thiyagarajan T, Tillmann H, Getzenberg RH and Narayanan R: Tissue selective androgen receptor modulators (SARMs) increase pelvic floor muscle mass in ovariectomized mice. J Cell Biochem 118: 640-646, 2017.

30. Chisamore MJ, Gentile MA, Dillon GM, Baran M, Gambone C, Riley S, Schmidt A, Flores O, Wilkinson H and Alves SE: A novel selective androgen receptor modulator (SARM) MK-4541 exerts anti-androgenic activity in the prostate cancer xenograft R-3327G and anabolic activity on skeletal muscle mass \& function in castrated mice. J Steroid Biochem Mol Biol 163: 88-97, 2016.

31. Blanqué R, Lepescheux L, Auberval M, Minet D, Merciris D, Cottereaux C, Clément-Lacroix P, Delerive P and Namour F: Characterization of GLPG0492, a selective androgen receptor modulator, in a mouse model of hindlimb immobilization. BMC Musculoslelet Disord 15: 291, 2014.

32. Akita K, Harada K, Ichihara J, Takata N, Takahashi Y and Saito K: A novel selective androgen receptor modulator, NEP28, is efficacious in muscle and brain without serious side effects on prostate. Eur J Pharmacol 720: 107-114, 2013.

33. Zhang X, Allan GF, Tannenbaum P, Sbriscia T, Linton O, Lai MT, Haynes-Johnson D, Bhattacharjee S, Lundeen SG and Sui Z: Pharmacological characterization of an imidazolopyrazole as novel selective androgen receptor modulator. J Steroid Biochem Mol Biol 134: 51-58, 2013.

34. Narayanan R, Mohler ML, Bohl CE, Miller DD and Dalton JT: Selective androgen receptor modulators in preclinical and clinical development. Nucl Recept Signal 6: e010, 2008.

35. Narayanan R, Coss CC, Yepuru M, Kearbey JD, Miller DD and Dalton JT: Steroidal androgens and nonsteroidal, tissue-selective androgen receptor modulator, S-22, regulate androgen receptor function through distinct genomic and nongenomic signaling pathways. Mol Endocrinol 22: 2448-2465, 2008.

36. Crawford J, Prado CM, Johnston MA, Gralla RJ, Taylor RP, Hancock ML and Dalton JT: Study design and rationale for phase 3 clinical development program of enobosarm, a selective androgen receptor modulator, for the prevention and treatment of muscle wasting in cancer patients (POWER Trials). Curr Oncol Rep 18: 37, 2016.

37. Garber K: No longer going to waste. Nat Biotechnol 34: 458-461, 2016. 